\title{
Once subtracted Roy-like dispersion relations and a precise analysis of $\pi \pi$ scattering data
}

\section{R. García-Martín* ${ }^{\dagger}$}

Dpto. Física Teórica II, Universidad Complutense, Madrid, Spain and

Groupe de Physique Théorique, Institut de Physique Nucléaire (IN2P3-CNRS), Orsay, France.

E-mail: garciadipno.in2p3.fr

\section{R. Kamiński}

Institute of Nuclear Physics, Polish Academy of Sciences, Cracow, Poland.

E-mail: Robert.Kaminski@ifj.edu.pl

\section{J. R. Peláez}

Dpto. Física Teórica II, Universidad Complutense, Madrid, Spain

E-mail: jrpelaezefis.ucm.es

\section{F. J. Ynduráin}

Departamento de Física Teórica C-XI, Universidad Autónoma de Madrid, Cantoblanco, Spain.

\begin{abstract}
We report our progress on the data analysis of $\pi \pi$ scattering data in terms of Forward Dispersion Relations (FDR), as well as Roy equations (RE) and their once-subtracted counterpart, GKPY equations. The first part of the analysis consists of independent fits to the different $\pi \pi$ channels. The GKPY equations provide a more stringent consistency check for the parametrizations of the $S 0$-wave data in the region from 400 to $1100 \mathrm{MeV}$, In the second part we present our preliminary analysis where the fits are constrained to satisfy all dispersion relations within errors, including the new GKPY Eqs., thus providing a very precise and model independent description of data using just analyticity, causality and crossing.
\end{abstract}

International Workshop on Effective Field Theories: from the pion to the upsilon February 2-6 2009

Valencia, Spain

\footnotetext{
*Speaker.

$\dagger$ We thank the organizers for creating the nice scientific atmosphere of the event and the Spanish research contracts PR27/05-13955-BSCH, FPA 2004-02602, UCM-CAM 910309 and BFM 2003-00856 for partial financial support. RGM's work is partially supported by the European commission MRTN FLAVIAnet [MRTN-CT-2006035482].
} 


\section{Introduction}

A precise knowledge of pion-pion scattering is of interest since it provides a test of Chiral Perturbation Theory (ChPT) as well as useful information about quark masses and the chiral condensate [1]. It is also of interest to establish the properties of the $f_{0}(600)$ or sigma meson (see talk by J. R. Peláez [3] in this conference and references therein). This reaction, at least in the elastic regime, is also remarkably symmetric in terms of isospin and crossing symmetries. Unfortunately, the existing experimental information on $\pi \pi$ scattering has many conflicting data sets at intermediate energies and used to have no data at all close to the interesting threshold region. For many years this fact has made it very hard to obtain conclusive results on $\pi \pi$ scattering at low energies or in the sigma region. However, recent [2] and precise experiments on kaon decays, related to $\pi \pi$ scattering at very low energies, have renewed the interest on this process.

The dispersive integral formalism is model independent, just based on analyticity and crossing, and relates the $\pi \pi$ amplitude at a given energy with an integral over the whole energy range, increasing the precision. It also provides information on the amplitude either at energies where data are poor or in the complex plane. In addition, it makes the parametrization of the data irrelevant once it is included in the integral and relates different scattering channels among themselves. For all these reasons it is well suited to study the threshold region or the poles in the complex plane associated to resonances. (For our progress and references on the latter, see [B] in this conference).

Our recent works make use of two complementary dispersive approaches, in brief:

Forward Dispersion Relations (FDRs) They are calculated at $t=0$ so that the unknown large-t behavior of the amplitude is not needed. We consider two symmetric and one asymmetric isospin combinations, to cover the isospin basis. The symmetric ones, $\pi^{0} \pi^{+}$and $\pi^{0} \pi^{0}$, have two subtractions and can be written as

$$
\operatorname{Re} F_{00,0+}(s, 0)-F_{00,0+}\left(4 M_{\pi}^{2}, 0\right)=\frac{s\left(s-4 M_{\pi}^{2}\right)}{\pi} \mathscr{P} . \mathscr{P} . \int_{4 M_{\pi}^{2}}^{\infty} d s^{\prime} \frac{\left(2 s^{\prime}-4 M_{\pi}^{2}\right) \operatorname{Im} F_{00,0+}\left(s^{\prime}, 0\right)}{s^{\prime}\left(s^{\prime}-s\right)\left(s^{\prime}-4 M_{\pi}^{2}\right)\left(s^{\prime}+s-4 M_{\pi}^{2}\right)}
$$

All contributions to their integrands are positive, which makes them very precise. The antisymmetric isospin combination $I_{t}=1$ does not require two subtractions:

$$
F^{\left(I_{t}=1\right)}(s, 0)=\frac{2 s-4 M_{\pi}^{2}}{\pi} \mathscr{P} . \mathscr{P} \cdot \int_{4 M_{\pi}^{2}}^{\infty} d s^{\prime} \frac{\operatorname{Im} F^{\left(I_{t}=1\right)}\left(s^{\prime}, 0\right)}{\left(s^{\prime}-s\right)\left(s^{\prime}+s-4 M_{\pi}^{2}\right)} .
$$

We have implemented all of them up to $\sqrt{s} \simeq 1420 \mathrm{MeV}$

Roy Equations (RE) [] An infinite set of coupled equations fully equivalent to nonforward dispersion relations, plus some $t-s$ crossing symmetry, written in terms of partial waves of definite isospin I and angular momentum $l$. The complicated left cut contribution is rewritten as series of integrals over partial waves in the physical region:

$$
\operatorname{Re} f_{\ell}^{(I)}(s)=C_{l}^{(I)} a_{0}^{(0)}+C_{\ell}^{(I)} a_{0}^{(2)}+\sum_{I^{\prime}, \ell^{\prime}} \mathscr{P} \cdot \mathscr{P} \cdot \int_{4 M_{\pi}^{2}}^{\infty} d s^{\prime} K_{\ell \ell^{\prime}}^{I I^{\prime}}\left(s^{\prime} ; s\right) \operatorname{Im} f_{\ell^{\prime}}^{\left(I^{\prime}\right)}\left(s^{\prime}\right)
$$

where the $C_{\ell}^{(I)}, C_{\ell}^{(I)}$ constants (actually, first order polynomials in $s$ ) and $K_{\ell \ell^{\prime}}^{I I^{\prime}}$ kernels are known. In practice, the calculation is truncated at $\ell<2$ and at some cutoff energy $s_{0}$. The $\ell \geq 2$ waves and 
the high energy are treated as input. RE are well suited to study poles of resonances but are limited to $\sqrt{s} \leq 8 M_{\pi} \simeq 1120 \mathrm{MeV}$. At present, we have implemented them up to $\sqrt{s} \simeq 2 m_{K}$.

In the last decade the use of RE has regained interest with several aims: to improve the precision of scattering data, to discard spurious solutions, to test ChPT, or to use ChPT to obtain the subtraction constants at low energies, which can be recast in terms of the scattering lengths $a_{0}^{(0)}$ and $a_{0}^{(2)}$, and to obtain precise predictions on $\pi \pi$ scattering. In addition it is of interest for the lightest scalar resonance, but that will be detailed in J.R. Peláez's talk in this conference [3]. In particular a series of RE analysis in [5] using $\pi \pi$ data parametrizations for the $\ell>2$ waves and above $800 \mathrm{MeV}$ for the rest, as well as some Regge input, was performed with and without ChPT constraints. The latter provided, $a_{0}^{(0)}=0.220 \pm 0.005 M_{\pi}^{-1}$ and $a_{0}^{(2)}=-0.0444 \pm 0.0010 M_{\pi}^{-1}$, an extremely precise claim, together with predictions for other scattering lengths and the $S$ - and $P$-wave phase shifts up to $800 \mathrm{MeV}$. Some of the input, particularly the Regge theory and the $D$ waves, was questionable [6], but it certainly seems to have a very small influence in the threshold region of the scalar waves [7]. Also in these period, the Krakow-Paris [8] and Paris [9] groups have performed other RE analysis. The former resolved the long-standing ambiguity, discarding the so-called "up"' solution, including in their analysis a study using polarized target data. The latter checked the calculation in [5] and claimed an small discrepancy in the Olsson sum rule. Recently, our group has also made a series of works on a dispersive analysis of $\pi \pi$ scattering that we review in the next section.

In section 3 we advance some results on our derivation of an additional set of Roy-like dispersive equations, which are obtained by starting with a once-subtracted dispersion relation. They impose additional constraints at intermediate energies, and allow us to reduce the uncertainty of the analysis.

\section{Overview of the analysis}

The approach we have followed throughout a series of works [10] can be summarized as follows:

1. We first obtain simple fits to data for each $\pi \pi$ scattering partial wave (the so called Unconstrained Fits to Data, or UFD08 for short). These fits are uncorrelated, therefore they can be very easily changed when new, more precise data become available. This has actually happened, for example, in [11], where we have included the newest, very precise $K_{l_{4}}$ data [2]. At different stages of our approach we have also fitted Regge theory to $\pi \pi$ high energy data, and as our precision was improving, we have improved some of the UFD fits with more flexible parametrizations.

2. Then, these UFD08 are checked against FDR, several sum rules, and Roy's Equations. Surprisingly, some widely used data parametrizations fail to satisfy the FDR or some of the sum rules. Thus we keep those parametrizations that are in better agreement with the FDR.

3. Finally, we impose these dispersive relations in the previous fits as additional constraints. These new Constrained Fits to Data (CFD08 for short) are much more precise and reliable than the UFD08 set, being consistent with analyticity, unitarity, crossing, etc. The price to pay is that now all the waves are correlated. 


\begin{tabular}{|c|c|c|c|c|}
\hline & \multicolumn{2}{|c|}{ Unconstrained Fits to Data (UFD08) } & \multicolumn{2}{|c|}{ Constrained Fits to Data (CFD08) } \\
\hline & $s^{1 / 2} \leq 932 \mathrm{MeV}$ & $s^{1 / 2} \leq 1420 \mathrm{MeV}$ & $s^{1 / 2} \leq 992 \mathrm{MeV}$ & $s^{1 / 2} \leq 1420 \mathrm{MeV}$ \\
\hline$\pi^{0} \pi^{0} \mathrm{FDR}$ & 0.12 & 0.29 & 0.13 & 0.31 \\
\hline$\pi^{+} \pi^{0}$ FDR & 0.84 & 0.86 & 0.83 & 0.85 \\
\hline$I_{t=1} \mathrm{FDR}$ & 0.66 & 1.87 & 0.13 & 0.70 \\
\hline & \multicolumn{2}{|c|}{$s^{1 / 2} \leq 992 \mathrm{MeV}$} & \multicolumn{2}{|c|}{$s^{1 / 2} \leq 992 \mathrm{MeV}$} \\
\hline Roy eq. S0 & \multicolumn{2}{|c|}{0.54} & \multicolumn{2}{|c|}{0.23} \\
\hline Roy eq. S2 & \multicolumn{2}{|c|}{1.63} & \multicolumn{2}{|c|}{0.25} \\
\hline Roy eq. P & \multicolumn{2}{|c|}{0.74} & \multicolumn{2}{|c|}{0.002} \\
\hline
\end{tabular}

Table 1: Average discrepancies $\vec{d}^{2}$ of the UFD08 and CFD08 for each FDR and RE. On average, the UFD are consistent with dispersion relations. Note the remarkable CFD08 consistency.

In order to quantify how well the dispersion relations are satisfied, we define six quantities $\Delta_{i}$ as the difference between the left and right sides of each dispersion relation in Eqs. (1.1), (1.2) and (1.3), whose uncertainties we call $\delta \Delta_{i}$. Next, we define the average discrepancies

$$
\bar{d}_{i}^{2} \equiv \frac{1}{\text { number of points }} \sum_{n}\left(\frac{\Delta_{i}\left(s_{n}\right)}{\delta \Delta_{i}\left(s_{n}\right)}\right)^{2}
$$

where the values of $s_{n}$ are taken at intervals of $25 \mathrm{MeV}$. Note the similarity with an averaged $\chi^{2} /($ d.o.f $)$ and thus $\vec{d}_{i}^{2} \leq 1$ implies fulfillment of the corresponding dispersion relation within errors. In Table 1 we show the average discrepancies of the UFD08 for each FDR, up to two different energies, and each RE up to $\sim 2 m_{K}$. Although the total average discrepancy of the UFD08 set is practically one, they can be clearly improved in the high energy region of the antisymmetric FDR and in the scalar isospin-2 RE. This is actually done in the CDF08 set, which is obtained by minimizing:

$$
\chi^{2} \equiv\left\{\vec{d}_{00}^{2}+\vec{d}_{0+}^{2}+\vec{d}_{I_{t}=1}^{2}+\vec{d}_{S 0}^{2}+\vec{d}_{S 2}^{2}+\vec{d}_{P}^{2}\right\} W+\vec{d}_{I}^{2}+\vec{d}_{J}^{2}+\sum_{i}\left(\frac{p_{i}-p_{i}^{\text {exp }}}{\delta p_{i}}\right)^{2}
$$

where $p_{i}^{\exp }$ are all the parameters of the different UFD08 parametrization for each wave or Regge trajectory, thus ensuring the data description, and $d_{I}$ and $d_{J}$ are the discrepancies for a couple of crossing sum rules. The weight $W=9$ was estimated from the typical number of degrees of freedom needed to describe the shape of the dispersion relations.

From the Table it is clear that the CFD set satisfies remarkably well all dispersion relations within uncertainties, and hence can be used directly and inside the Olsson sum rule to obtain the following precise determination from data: $a_{0}^{(0)}=0.223 \pm 0.009 m_{\pi}^{-1}$ and $a_{0}^{(2)}=-0.0444 \pm$ $0.0045 m_{\pi}^{-1}$. This is in remarkable agreement with the predictions of RE and ChPT of [5]. Nevertheless, the agreement is fairly good only up to roughly $450 \mathrm{MeV}$, but from that energy up to 800 $\mathrm{MeV}$ those predictions deviate from our data analysis. We should stress that we are nevertheless talking about a disagreement of a few degrees at most and would affect the determination of the sigma mass by tens of $\mathrm{MeV}$ at most, which is a remarkable improvement compared with the situation just a few years ago and the huge and extremely conservative uncertainties quoted in the PDG for the $\sigma$ mass and width, of hundreds of $\mathrm{MeV}$. 
The other waves are of less relevance for this conference and we comment them very briefly, since the details can be found in [10]. The best determination of threshold parameters is obtained by using the CFD08 set inside appropriate sum rules [10]. In brief, we agree with [5] in the $P$-wave scattering length, but find disagreements of 2 to 3 standard deviations in the P-wave slope, and also in some $D$-wave parameters.

In summary the CFD08 set provides a model independent and very precise description of the $\pi \pi$ scattering data consistent with analyticity and crossing.

\section{Work in progress: GKPY equations and 400 to $1100 \mathrm{MeV}$ region}

The precision of the analysis sketched in the previous section in the $\sqrt{s}>400 \mathrm{MeV}$ region is limited by the big uncertainties that Roy's equations propagate at high energies, in particular those coming from the experimental uncertainty in $a_{0}^{(2)}$. By starting with a once-subtracted dispersion relation we arrive at the following set of Roy-like equations (GKPY for brevity):

$$
\operatorname{Re} f_{\ell}^{(I)}(s)=D_{\ell}^{(I)}+\sum_{I^{\prime}, \ell^{\prime}} \mathscr{P} \cdot \mathscr{P} \cdot \int_{4 M_{\pi}^{2}}^{\infty} d s^{\prime} \tilde{K}_{\ell \ell^{\prime}}^{I I^{\prime}}\left(s^{\prime} ; s\right) \operatorname{Im} f_{\ell^{\prime}}^{\left(I^{\prime}\right)}\left(s^{\prime}\right) .
$$

in which the $\tilde{K}_{\ell \ell^{\prime}}^{I I^{\prime}}$ kernels and $D_{\ell}^{(I)}$ subtraction constant are known and will be explicitely given in an upcoming work. It is important to note that, whereas the subtraction constants in RE constitute a first-order polynomial in $s$, they are here just combinations of $a_{0}^{(0)}$ and $a_{0}^{(2)}$. Therefore, the propagation of uncertainties (see Fig. 1) is as follows: for $\sqrt{s}<400 \mathrm{MeV}$ RE have smaller uncertainties, but for $\sqrt{s}>450 \mathrm{MeV}$ GKPY equations have a significantly smaller error band. This enables further improvements in our fits for the $S 0$-wave in the 400 to $1100 \mathrm{MeV}$ region. In fact, the level of precision attained in this region forces us to refine our fits by means of an improved matching between the $S 0$-wave parametrizations at low and intermediate energies, which occurs at $932 \mathrm{MeV}$, imposing also continuity in the first derivative. Preliminary results for the phase shifts can be seen in Fig. 2 (left), to compare with that of the previous fit (dashed line). For clarity, we do not provide the data points, which are nevertheless reasonably well described by both parametrizations when taking into account the experimental errors.

Finally, we have obtained a new CFD using this improved UFD as the starting point, and adding the GKPY equations as an additional constraint. The results obtained (see plot on the right in Fig. 2) are very similar to the previous CFD, but GKPY fulfillment is improved.

\section{Acknowledgments}

This work is dedicated to the memory of Prof. Ynduráin who recently passed away but participated decisively in the main discussions and initial calculations of this work.

\section{References}

[1] J. Gasser and H. Leutwyler, Annals Phys. 158, 142 (1984).

[2] J. R. Batley et al. [NA48/2 Collaboration], Eur. Phys. J. C 54 (2008) 411. S. Pislak et al. [BNL-E865 Collaboration], Phys. Rev. Lett. 87 (2001) 221801 [arXiv:hep-ex/0106071]. 

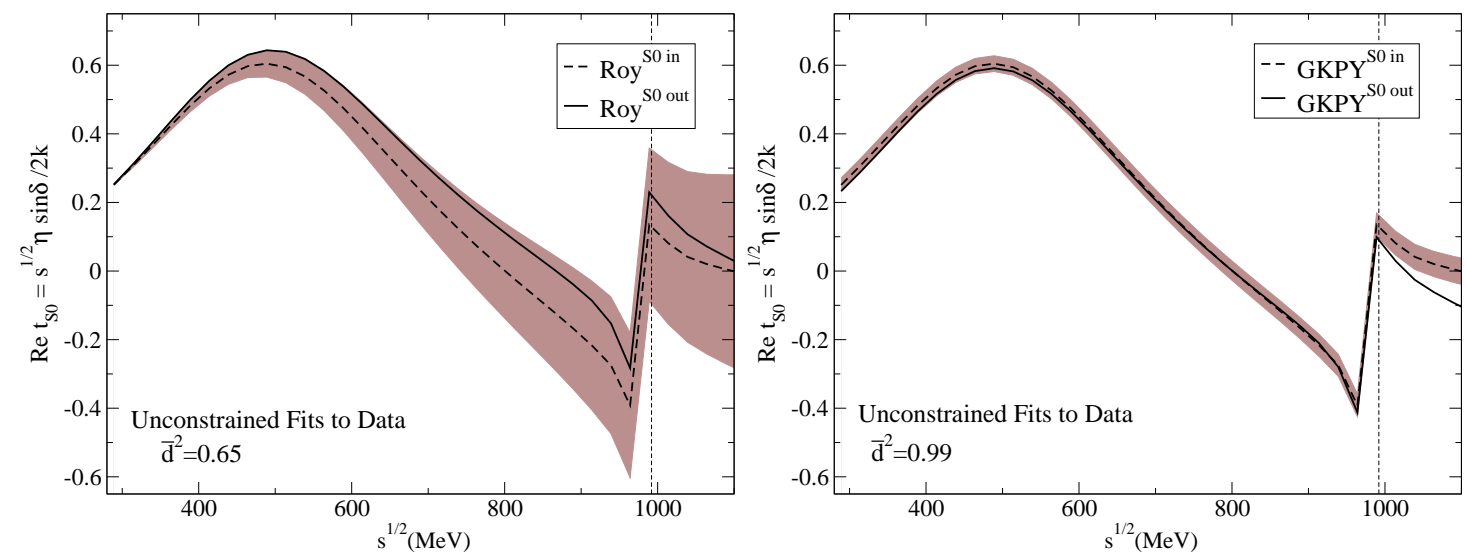

Figure 1: Above $450 \mathrm{MeV}$ the uncertainty in the standard $S 0$-wave Roy eqs. (left) is much larger than in the once-subtracted GKPY equations (right). We show results for the new UFD S0 wave parametrization.
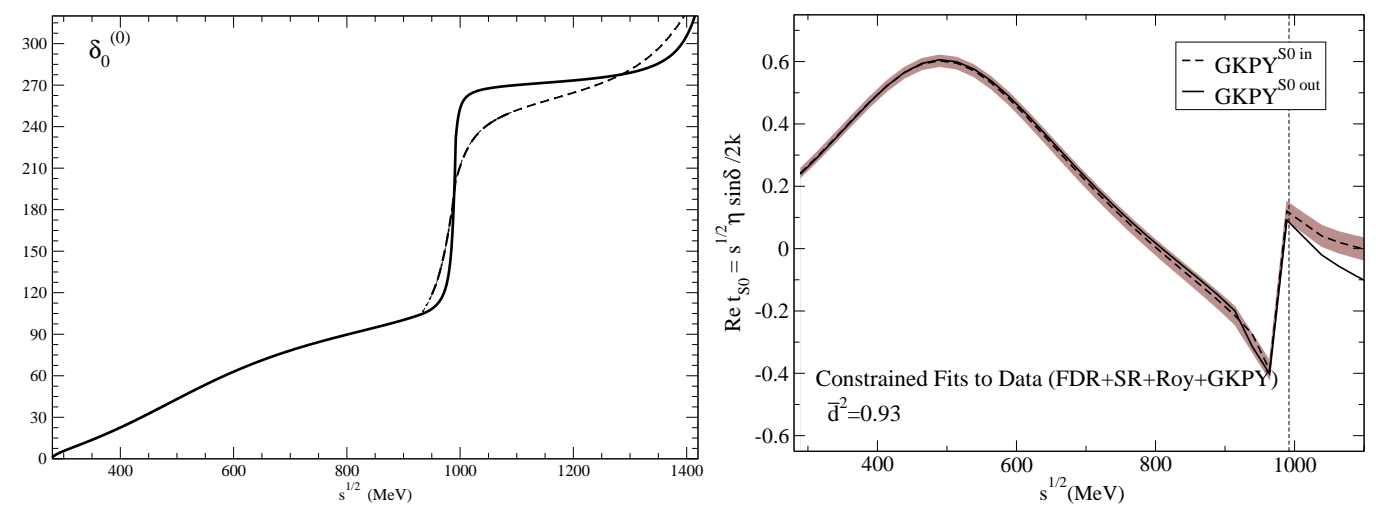

Figure 2: Left: The improved matching only changes the $S 0$-wave above $932 \mathrm{MeV}$. Right: agreement with GKPY eqs. is improved above $850 \mathrm{MeV}$ in the preliminary CFD with improved matching and GKPY.

[3] J. R. Peláez, R. Garcia-Martin and R. Kaminski, on this conference.

[4] S. M. Roy, Phys. Lett. B 36, 353 (1971).

[5] G. Colangelo, J. Gasser and H. Leutwyler, Nucl. Phys. B 603 (2001) 125 B. Ananthanarayan, G. Colangelo, J. Gasser and H. Leutwyler, Phys. Rept. 353 (2001) 207

[6] J. R. Pelaez and F. J. Yndurain, Nucl. Phys. Proc. Suppl. 164 (2007) 93; Phys. Rev. D 68 (2003) 074005

[7] I. Caprini, G. Colangelo, J. Gasser and H. Leutwyler, Phys. Rev. D 68 (2003) 074006

[8] R. Kaminski, L. Lesniak and B. Loiseau, Phys. Lett. B 551, 241 (2003)

[9] S. Descotes-Genon, N. H. Fuchs, L. Girlanda and J. Stern, Eur. Phys. J. C 24, 469 (2002)

[10] R. Kaminski, J. R. Pelaez and F. J. Yndurain, Phys. Rev. D 77 (2008) 054015; Phys. Rev. D 74 (2006) 014001

[11] R. Garcia-Martin and J. R. Pelaez, F. J. Yndurain, Phys. Rev. D 76 (2007) 074034 J. R. Pelaez and F. J. Yndurain, Phys. Rev. D 71 (2005) 074016; Phys. Rev. D 69 (2004) 114001 